\title{
A Network of Psychopathological, Cognitive, and Motor Symptoms in Schizophrenia Spectrum Disorders
}

Citation for published version (APA):

Moura, B. M., Van Rooijen, G., Schirmbeck, F., Wigman, H., Madeira, L., Van Harten, P., Van Os, J., Bakker, P. R., Marcelis, M., Genetic Risk and Outcome of Psychosis (GROUP) Investigators, van Amelsvoort, T., \& Simons, C. (2021). A Network of Psychopathological, Cognitive, and Motor Symptoms in Schizophrenia Spectrum Disorders. Schizophrenia Bulletin, 47(4), 915-926.

https://doi.org/10.1093/schbul/sbab002

Document status and date:

Published: 01/07/2021

DOI:

10.1093/schbul/sbab002

Document Version:

Publisher's PDF, also known as Version of record

\section{Document license:}

Taverne

Please check the document version of this publication:

- A submitted manuscript is the version of the article upon submission and before peer-review. There can be important differences between the submitted version and the official published version of record.

People interested in the research are advised to contact the author for the final version of the publication, or visit the DOI to the publisher's website.

- The final author version and the galley proof are versions of the publication after peer review.

- The final published version features the final layout of the paper including the volume, issue and page numbers.

Link to publication

\footnotetext{
General rights rights.

- You may freely distribute the URL identifying the publication in the public portal. please follow below link for the End User Agreement:

www.umlib.nl/taverne-license

Take down policy

If you believe that this document breaches copyright please contact us at:

repository@maastrichtuniversity.nl

providing details and we will investigate your claim.
}

Copyright and moral rights for the publications made accessible in the public portal are retained by the authors and/or other copyright owners and it is a condition of accessing publications that users recognise and abide by the legal requirements associated with these

- Users may download and print one copy of any publication from the public portal for the purpose of private study or research.

- You may not further distribute the material or use it for any profit-making activity or commercial gain

If the publication is distributed under the terms of Article $25 \mathrm{fa}$ of the Dutch Copyright Act, indicated by the "Taverne" license above, 


\title{
A Network of Psychopathological, Cognitive, and Motor Symptoms in Schizophrenia Spectrum Disorders
}

\begin{abstract}
Bernardo Melo Moura*,1-3, Geeske van Rooijen ${ }^{4}$, Frederike Schirmbeck ${ }^{4,5}$, Johanna T. W. Wigman ${ }^{7}$, Genetic Risk and Outcome of Psychosis (GROUP) investigators, Luís Madeira' ${ }^{1,2}$, Peter van Harten ${ }^{3,8}$, Jim van Os ${ }^{3,9}$, P. Roberto Bakker ${ }^{3,5,9}$, and Machteld Marcelis ${ }^{3,10}$

${ }^{1}$ Department of Psychiatry, Faculty of Medicine, University of Lisbon, 1649-028 Lisbon, Portugal; ${ }^{2}$ Department of Psychiatry and Mental Health, North Lisbon University Hospital Centre, Avenida Professor Egas Moniz, 1649-028 Lisbon, Portugal; ${ }^{3}$ Department of Psychiatry and Neuropsychology, School for Mental Health and Neuroscience, Maastricht University Medical Centre, Maastricht University, 6200 MD Maastricht, The Netherlands; ${ }^{4}$ Department of Psychiatry, Academic Medical Center, University of Amsterdam, 1105 AZ Amsterdam, The Netherlands; ${ }^{5}$ Arkin Institute for Mental Health, 1033 NN Amsterdam, The Netherlands; ${ }^{6}$ Interdisciplinary Center Psychopathology and Emotion Regulation, University Medical Center Groningen, University of Groningen, 9713 GZ

Groningen, The Netherlands; ${ }^{7}$ Rob Giel Onderzoekscentrum, University Medical Center Groningen, University of Groningen, 9713 GZ Groningen, The Netherlands; ${ }^{8}$ GGz Centraal, Innova Medical Centre, 3800 DB Amersfoort, The Netherlands; ${ }^{9}$ Brain Center Rudolf Magnus University Medical Center Utrecht, Utrecht University, 3508 AB Utrecht, The Netherlands; ${ }^{10}$ Institute for Mental Health Care Eindhoven (GGzE), 5600 AX Eindhoven, The Netherlands
\end{abstract}

*To whom correspondence should be addressed; tel: +351 217805 000; fax: +351 217805 610; e-mail: bernardomoura@campus.ul.pt The Genetic Risk and Outcome of Psychosis (GROUP) investigators are listed above the References section.

Schizophrenia spectrum disorders (SSDs) are complex syndromes involving psychopathological, cognitive, and also motor symptoms as core features. A better understanding of how these symptoms mutually impact each other could translate into diagnostic, prognostic, and, eventually, treatment advancements. The present study aimed to: (1) estimate a network model of psychopathological, cognitive, and motor symptoms in SSD; (2) detect communities and explore the connectivity and relative importance of variables within the network; and (3) explore differences in subsample networks according to remission status. A sample of 1007 patients from a multisite cohort study was included in the analysis. We estimated a network of $\mathbf{4 3}$ nodes, including all the items from the Positive and Negative Syndrome Scale, a cognitive assessment battery and clinical ratings of extrapyramidal symptoms. Methodologies specific to network analysis were employed to address the study's aims. The estimated network for the total sample was densely interconnected and organized into 7 communities. Nodes related to insight, abstraction capacity, attention, and suspiciousness were the main bridges between network communities. The estimated network for the subgroup of patients in remission showed a sparser density and a different structure compared to the network of nonremitted patients. In conclusion, the present study conveys a detailed characterization of the interrelations between a set of core clinical elements of SSD. These results provide potential novel clues for clinical assessment and intervention.
Key words: network analysis/psychosis/positive symptoms/negative symptoms/cognition/extrapyramidal symptoms

\section{Introduction}

Schizophrenia spectrum disorders (SSDs) are complex syndromes with widely different clinical presentations. ${ }^{1}$ Psychopathological symptoms are the most visible manifestations and, therefore, used to guide much research. However, 2 other domains - cognitive and motor symptoms - are also impactful. Impairments in social cognition and neurocognition are associated with worse functioning and overall prognosis while also being closely tied to psychopathological symptoms. ${ }^{2,3}$ Motor abnormalities are increasingly regarded as a crucial element of SSD not only as side effects of antipsychotic medication but also as having diagnostic and prognostic value early in the course of illness. ${ }^{4,5}$ Extrapyramidal symptoms (EPS), in particular, are a common motor abnormality that is associated with cognitive deficits already in the early stages of psychotic disorders. ${ }^{6}$ Thus, psychopathological, cognitive, and motor symptoms are interrelated in complex ways in the clinical picture of SSD - possibly reflecting common neurobiological underpinnings ${ }^{7}$ - requiring more investigation. A better understanding of how these elements mutually impact each other may 
reveal novel insights into the diagnosis, prognosis, and treatment of SSD.

Network models may form a suitable approach to jointly study these areas of clinical manifestations. ${ }^{8,9}$ Network analysis as a tool offers a way to overcome some limitations of other analytical methods that deal with complex interdependencies (eg, need for a priori assumptions in structural equation models or absence of feedback loops in directed acyclical graphs) and generates specific metrics (eg, centrality), which might enrich our knowledge about mental disorders. ${ }^{10}$ Moreover, this approach paves the way for a network theory of mental disorders, which asserts that mutually impacting symptoms regulate mental functioning rather than psychological latent constructs. This approach is also aligned with the recent theory that considers health and disease as states of systems that can be studied as networks at multiple levels. ${ }^{11}$

The most used method for network analysis in psychology research is the Gaussian Graphical Model (GGM), extensively applied to cross-sectional data. ${ }^{12}$ In this model, a connection (edge) between 2 variables (nodes) is depicted if they show a pattern of co-occurrence across cases. An edge between 2 nodes can be interpreted as indicative of a potential causal relation..$^{13}$ Through GGM, one can explore a set of clinically relevant variables and gain further insight into, most notably, which elements are crucial in sustaining the network's local and global structure (nodes acting as hubs or bridges between domains are particularly relevant). In less technical terms, this means identifying which symptoms might be responsible for keeping pathological states active.

Several cross-sectional studies used network analysis to investigate clinical variables in psychotic disorders ${ }^{14-16}$; however, to our knowledge, only 3 included the combination of psychopathological (factor level) and cognitive symptoms. ${ }^{17-19}$ Overall, no previous research looked simultaneously at psychopathology, cognition, and motor symptoms.

We aimed: (1) to estimate a network consisting of psychopathological, cognitive, and motor symptoms in patients with SSD; (2) to describe the network structure by identifying the most important nodes in the overall network and in connecting different variable domains; and (3) to explore network differences between patients in remission vs patients not in remission, given that SSDs frequently are persistent disorders that greatly vary in their clinical presentation according to remission status (in psychotic episodes or between episodes).

\section{Methods}

\section{Subjects}

In the current study, data collected in the Genetic Risk and Outcome of Psychosis (GROUP) study were used. The GROUP study is a multisite longitudinal cohort investigation run across 4 academic centers in the Netherlands (Amsterdam, Groningen, Maastricht, and Utrecht) and including data from patients, siblings, parents, and controls. It is described extensively elsewhere. ${ }^{20}$ For the current study, only baseline patient data were considered (collected between 2004 and 2007). All the participants had a diagnosis of a nonaffective psychotic disorder according to the Diagnostic and Statistical Manual of Mental Disorders, Fourth Edition, criteria ${ }^{21}$ and were aged between 16 and 50 years.

\section{Assessments}

Over 30 interviewers per recruitment site were trained for administering the assessments. The Positive and Negative Syndrome Scale (PANSS) was used to assess psychopathological symptoms. ${ }^{22}$ This instrument is composed of 30 items distributed over 3 subscales: positive, negative, and general psychopathology. The PANSS total score had an intraclass correlation coefficient of 0.946 (95\% CI: 0.758-0.996), thus the interrater reliability was considered good.

A battery of neuropsychological tests was used. It covered the fundamental domains affected in schizophrenia as considered in the MATRICS consensus, ${ }^{23}$ with the exception of visual learning and memory (not included in the GROUP study): processing speed-Digit Symbol Substitution Test from the Wechsler Adult Intelligence Scale-III (WAIS-III) 24 ; working memory-arithmetic subtest from the WAIS-III; reasoning and problem solving - Response Set-shifting Task, ${ }^{25}$ accuracy cost score (RST), and the block design subtest from the WAIS-III; verbal learning and memory - Word Learning Task, ${ }^{26}$ correct delayed recall items; attention/vigilance - Continuous Performance Test- $\mathrm{HQ}^{27}$, number of correct positives; social cognition-Benton Facial Recognition Test, ${ }^{28}$ total correct items, Degraded Facial Affect Recognition Test, ${ }^{29}$ percentage of correct items (DFAR), and the Hinting task, ${ }^{30}$ total score. The WAIS-III information subtest was also included as a general knowledge assessment.

Motor symptoms were assessed with the following scales for different EPS domains: Unified Parkinson's Disease Rating Scale, ${ }^{31}$ motor examination section score (UPDRS); Barnes Akathisia Rating Scale, ${ }^{32}$ global clinical assessment of akathisia; a rating of dystonia; Abnormal Involuntary Movement Scale, ${ }^{33}$ overall score (AIMS). Akathisia and dystonia were graded on an ordinal scale, respectively, from 0 (absent) to 5 (severe) and from 0 (absent) to 4 (severe). All other measures in the analysis were considered continuous.

Subjects with missing data represented $30.1 \%$ of the total sample (315/1047). We opted to use a conservative approach and excluded all cases with more than $15 \%$ missing values $(N=40)$. Multiple imputation was used for all other cases (mice package for $\mathrm{R}^{34}$; supplementary figure $\mathrm{S} 1$ ). 


\section{Network Analysis}

Network Estimation A nonparanormal transformation using the huge package was applied to all the included variables, except the ordinal variables akathisia and dystonia, in order to relax the normality assumption..$^{35}$ This step also transformed values through standardization.

A network with 43 nodes was constructed using a GGM and implemented through the R statistical software version 3.6.2. ${ }^{36}$ with the packages bootnet and qgraph. ${ }^{37,38}$ Edges between nodes were undirected (no arrowhead) and established while controlling for the influence of every other node in the network. Furthermore, a L1-regularization procedure was used-Graphical Least Absolute Shrinkage and Selection Operator (GLASSO) - in combination with Extended Bayesian Information Criterion (EBIC). GLASSO limits spurious connections between nodes (false-positives) by shrinking small correlations to 0 . The EBIC is a goodness-offit measure for model selection that is tuned through a hyperparameter $\gamma$. Following recommendations in the field, this hyperparameter was tuned to 0.5 , ensuring a good balance between sensitivity and specificity. ${ }^{39}$ Polyserial partial correlations were calculated between the ordinal and the continuous variables; Pearson partial correlations were used in all other cases. The network layout followed the Fruchterman-Reingold algorithm, a method that places strongly related nodes close to each other and repels less related nodes. ${ }^{40}$ In this layout, the edges display their strength (edge-weight) through line thickness and direction through color (blue for positive partial correlations and dashed red for negative ones).

Stability and Accuracy Checks Following recommendations in this field, bootstrapping routines were used to assess the estimated model's stability and accuracy. ${ }^{13}$ Further details about these methods can be found as supplementary material.

Community Detection An Exploratory Graph Analysis (EGA) implemented through the package EGAnet was used to investigate how the network was organized in terms of dimensions. ${ }^{41}$ This method is based on the GLASSO and uses a random walk algorithm to identify communities in the network. The stability of the EGA's estimation was also assessed through bootstrapping the empirical correlation matrix with 1000 iterations. ${ }^{42}$ This approach allows to quantify the stability of each node regarding their community placement.

Network Metrics-Small-Worldness Index and Centrality Analysis The small-worldness index quantifies how much a network displays small-world properties (qgraph package)..$^{43}$ In a small-world network, there is a balance between local robustness of connections (high clustering coefficient) and a short distance between any pair of nodes (short path length). In the context of mental disorders, this means that certain symptoms will be more closely tied and reinforcing each other. They will thus segregate into communities representing syndromes (eg, negative symptoms) bound to each other by "bridge" symptoms.

Two different types of centrality measures were calculated for each node. Strength centrality measures the number of connections of a node considering their weight; it represents the influence of a node considering the local structure of the network (ie, its direct connections with other symptoms). This measure seems to be robust, predictive of subsequent diagnosis in longitudinal studies, and indicative of potential to change the remainder network as reviewed by Robinaugh et al. ${ }^{12}$ Bridge strength centrality (networktools package ${ }^{44}$ ) measures the weighted connectivity of a node in a given community to other communities in a network ${ }^{45}$ Communities are sets of highly interconnected nodes in a network. In the medical setting, they might represent clinical syndromes or variable domains. For this study, communities were established through a data-driven approach described in 2.3.3. Network Comparison Test: Remitted vs. Nonremitted Patients A binary division of the sample was carried out according to remission status (see supplementary table S3) ${ }^{46}$ A network was estimated for each subsample (see 2.3.1), and a Network Comparison Test (NCT) between the networks was performed. ${ }^{47}$ The NCT (NetworkComparisonTest package) uses a permutation approach to test for differences between the networks' invariant structure and invariant global strength. Moreover, we calculated strength centrality for each node of each subsample's network. We also carried out a stability analysis for each of the subsample's networks and tested for differences between clinical and sociodemographic variables.

Sensitivity Analysis An NCT was performed between the complete sample network and a network estimated without the patients taking typical antipsychotics. This was done to probe the influence on the network's connectivity of patients taking antipsychotics that are more EPS inducing.

\section{Results}

\section{Sample Characteristics}

Table 1 displays the main demographic and clinical characteristics of the included patients $(N=1007$; see supplementary table $\mathrm{S} 1$ for a detailed description by the diagnostic group). Table 2 shows the mean score and SD for each variable included as a node in the estimated network. The dystonia score distribution was extremely skewed (96\% of subjects rated with absent); therefore, this variable was discarded. The akathisia score was compressed to only vary between 0 and 2 due to few cases with higher scores (scores 3 and 4 converted to 2; no cases scored 5). The 2 subsamples defined according to remission status were composed of 451 patients in remission 
Table 1. Demographic and clinical characteristics of the included sample

\begin{tabular}{lc}
\hline Variable & Participants $(N=1007)$ \\
\hline Age, mean (SD), years & $27.3(7.4)$ \\
Females, no. (\%) & $231(22.9)$ \\
Education, highest achieved & $6(<1)$ \\
$\quad$ No education, no. (\%) & $130(13)$ \\
Elementary, no. (\%) & $563(56.4)$ \\
Secondary, no. (\%) & $299(30.0)$ \\
University or vocational, no. (\%) & $88 / 993(8.9)$ \\
Married, no. (\%) & $485 / 866(56.0)$ \\
Working (full time or part time), no. $(\%)$ & $5.0(4.4)$ \\
Illness duration, mean (SD), years & $51.6(15.9)$ \\
Global assessment of functioning, mean & \\
(SD) & $872 / 876(99.5)$ \\
Use of antipsychotic medication, no. & \\
(\% yes) & $104 / 872(11.9)$ \\
Use of typical antipsychotics, no. $(\%)$ & \\
Diagnosis & $668(66.5)$ \\
Schizophrenia, no. (\%) & $118(11.7)$ \\
Schizoaffective disorder, no. $(\%)$ & $102(10.1)$ \\
Psychosis NOS, no. (\%) & $56(5.6)$ \\
Schizophreniform disorder, no. $(\%)$ & $20(2.0)$ \\
Brief psychotic disorder, no. $(\%)$ & $41(4.1)$ \\
Other diagnosis, no. (\%) & $451 / 997(45.2)$ \\
In clinical remission, no (\%) & \\
\hline
\end{tabular}

Note: NOS, not otherwise specified.

and 546 not in remission (10 subjects lacked information on remission status).

\section{Network Analysis}

Figure 1 displays the estimated network. For the partial correlation matrix used as input, see supplementary table S2. The estimated network was highly interconnected and every node displayed edges to other nodes.

Stability and Accuracy Checks The bootstrapping routines employed to assess the stability and accuracy of strength centrality and edge weights returned satisfactory results (complete analysis available as supplementary figures S2-S5).

Community Detection Seven communities were found with the EGA and confirmed through parametric bootstrapping (figure 1). All the cognitive nodes belonged to one single community, which also encompassed "difficulty in abstract thinking" (N5). The remaining items from the PANSS formed 5 communities. All 3 motor nodes composed a separate single community. Node stability regarding community placement can be retrieved in supplementary figure S6. Notable cases of unstable placement were the nodes P4, P7, G8, G14 (together composing one community), and N5.

Small-Worldness Index and Centrality Analysis The smallworldness index was 1.46, above the threshold value of 1 set by Humphries and Gurney ${ }^{43}$; hence, the network can be considered to have small-world properties.
Figure 2 displays the different centrality measures per node ( $z$-transformed). The node with the highest strength centrality score was distinctively "delusions" (P1). Cognitive nodes belonging to the WAIS were in the upper half of strength centrality scores and the other cognitive nodes were in the bottom half. Parkinsonism node (UPDRS) presented the highest strength centrality for a motor node. Regarding bridge strength centrality, the strongest bridges for each community were "lack of judgment and insight" (G12), "suspiciousness" (P6), "difficulty in abstract thinking" (N5), "excitement" (P4), "motor retardation" (G7), "tension" (G4), and parkinsonism (UPDRS).

NCT: Remitted vs Nonremitted Patients The remitted patients' network was sparser, even presenting 3 disconnected nodes (DFAR, RST, and AIMS; figure 3A). The comparison test between the networks of patients in remission vs nonremitters yielded significant results for differences in global strength $(P<.01)$ and network structure $(P=.012)$. Figure 3B shows strength centrality for nodes of both subsample networks. Certain nodes changed widely their strength centrality according to remission status, especially "Excitement" (P4), "Emotional withdrawal" (N2), and theory of mind (Hinting task).

Stability analysis of the subsample networks rendered less stable results when compared to the full network, in particular for the network of patients in remission (supplementary figures S9-S12). Clinical and demographic features varied significantly between the 2 subsamples (supplementary table S4).

Sensitivity Analysis No significant differences were found between the network of patients not taking typical antipsychotics and the full-sample network (see supplementary figure $\mathrm{S} 11$ ).

\section{Discussion}

To our knowledge, this is the first network analysis study examining associations between psychopathology, cognition, and motor variables in patients with SSD. Overall, our results confirmed that these 3 clinical domains are highly interrelated but, at the same time, organized into separate communities. These communities followed, in general, the expected factor organization of the variables. Nodes related to insight, abstraction capacity, attention, and suspiciousness were the main bridges between network communities. Lastly, different clinical states were represented by different network architectures.

\section{Community Detection}

Within the PANSS items, our data-driven network approach replicated the most consensual 5-factor solution-positive, negative, disorganized, excited, and depressed/emotional distress factors. ${ }^{48}$ The main difference found was that "difficulty in abstract thinking" (N5) 
Table 2. Descriptive statistics of the variables submitted to network analysis

\begin{tabular}{|c|c|}
\hline Domains and variables & Mean (SD) \\
\hline \multicolumn{2}{|l|}{ Positive symptoms } \\
\hline P1-Delusions & $2.41(1.52)$ \\
\hline P2-Conceptual disorganization & $1.69(1.05)$ \\
\hline P3-Hallucinations & $2.11(1.53)$ \\
\hline P4-Excitement & $1.33(0.76)$ \\
\hline P5-Grandiosity & $1.50(1.00)$ \\
\hline P6-Suspiciousness/persecution & $2.27(1.41)$ \\
\hline P7-Hostility & $1.32(0.77)$ \\
\hline \multicolumn{2}{|l|}{ Negative symptoms } \\
\hline N1-Blunted affect & $2.35(1.33)$ \\
\hline N2-Emotional withdrawal & $2.13(1.20)$ \\
\hline N3-Poor rapport & $1.72(1.02)$ \\
\hline N4-Passive/apathetic social withdrawal & $2.27(1.31)$ \\
\hline N5-Difficulty in abstract thinking & $2.02(1.28)$ \\
\hline N6-Lack of spontaneity and flow of conversation & $1.90(1.20)$ \\
\hline N7-Stereotyped thinking & $1.59(0.96)$ \\
\hline \multicolumn{2}{|l|}{ General psychopathology } \\
\hline G1-Somatic concern & $1.68(1.81)$ \\
\hline G2-Anxiety & $2.34(1.27)$ \\
\hline G3 - Guilt feelings & $1.97(1.25)$ \\
\hline G4-Tension & $1.84(1.03)$ \\
\hline G5-Mannerisms and posturing & $1.30(0.72)$ \\
\hline G6-Depression & $2.47(1.41)$ \\
\hline G7-Motor retardation & $1.86(1.11)$ \\
\hline G8-Uncooperativeness & $1.23(0.63)$ \\
\hline G9-Unusual thought content & $2.04(1.31)$ \\
\hline G10-Disorientation & $1.19(0.56)$ \\
\hline G11-Poor attention & $1.73(1.02)$ \\
\hline G12 - Lack of judgment and insight & $2.10(1.39)$ \\
\hline G13-Disturbance of volition & $1.59(0.93)$ \\
\hline G14-Poor impulse control & $1.31(0.74)$ \\
\hline G15-Preoccupation & $1.47(0.89)$ \\
\hline G16-Active social avoidance & $1.76(1.08)$ \\
\hline \multicolumn{2}{|l|}{ Motor symptoms } \\
\hline Parkinsonism (Unified Parkinson's Disease Rating & $0.21(0.26)$ \\
\hline \multicolumn{2}{|l|}{ Scale) } \\
\hline Dyskinesia (Abnormal Involuntary Move & $0.07(0.18)$ \\
\hline \multicolumn{2}{|l|}{ Akathisia (Barnes Akathisia Rating Scale) } \\
\hline 0 (absent) & $796(79 \%)$ \\
\hline 1 (questionable) & $109(11 \%)$ \\
\hline 2 (mild or more severe) & $102(10 \%)$ \\
\hline \multicolumn{2}{|l|}{ Cognition } \\
\hline Processing speed: WAIS-III Digit Symbol Substitution & $7.41(3.01)$ \\
\hline \multicolumn{2}{|l|}{ Test } \\
\hline World knowledge: WAIS-III Information & $10.65(3.09)$ \\
\hline Working memory: WAIS-III Arithmetic & $9.50(3.26)$ \\
\hline $\begin{array}{l}\text { Reasoning and problem solving: WAIS-III Block de- } \\
\text { sign }\end{array}$ & $9.60(3.36)$ \\
\hline $\begin{array}{l}\text { Verbal learning and memory: Word Learning Task, } \\
\text { delayed recall }\end{array}$ & $7.54(2.88)$ \\
\hline Attention/vigilance: Continuous Performance Task & $26.43 / 28(2.77)$ \\
\hline \multicolumn{2}{|l|}{ HQ } \\
\hline Reasoning and problem solving: Response Set-shifting & $0.20(0.27)$ \\
\hline \multicolumn{2}{|l|}{ Task $^{\mathrm{a}}$} \\
\hline $\begin{array}{l}\text { Social cognition (emotional face recognition): De- } \\
\text { graded Facial Affect Recognition Test }\end{array}$ & $68.76(10.53)$ \\
\hline Social cognition (facial recognition): Benton Facial & $22.77(2.29)$ \\
\hline \multicolumn{2}{|l|}{ Recognition Test } \\
\hline Social cognition (theory of mind): hinting task & $17.52(2.79)$ \\
\hline
\end{tabular}

${ }^{\text {a }}$ Scores were transformed to the symmetrical distribution so that they could have the same meaning as the other cognitive variables (ie, higher score means better performance).

was part of the cognitive community in the current study, whereas it is commonly placed with disorganized symptoms. This was probably due to the simultaneous analysis of cognitive measures in the present study and not strictly the PANSS as in the factor analytic studies.

As expected, cognitive and motor variables formed 2 separate communities. The relatively small number of nodes in each of these domains might explain why no further communities were detected. Some nodes, notably N5 and the excitement community, had unstable community placements, probably reflecting multidimensionality (supplementary figure S6).

\section{Small-Worldness Index and Centrality Analysis}

The estimated network showed high interconnectivity, also extending the finding of small-world properties of psychopathological networks to a broader set of symptoms. ${ }^{49}$ This means that interferences at any node/edge within the network will likely affect many other nodes/ edges directly or indirectly within a few steps. At the same time, some nodes might play a more important role in network connectivity, acting as bridges between communities.

Within each of the 5 communities composed of PANSS items, the node with highest strength centrality corresponded to the item that commonly has the highest factor loading-P1 for positive symptoms, N6 for negative symptoms, $\mathrm{P} 2$ for disorganization, $\mathrm{P} 4$ for excitement, and $\mathrm{G} 2$ for emotional distress. ${ }^{50}$ This is in line with recent insights in the field that state that strength centrality is redundant with factor loading. ${ }^{51}$ Results from previous network analysis studies using the PANSS were largely replicated, except for N6 (alogia factor), which had been reported as a less central node. ${ }^{52,53} \mathrm{~A}$ possible explanation for this difference concerns the high amount of connections N6 establishes outside of the negative symptom domain, making it more central in the present study. The same pattern occurred for cognition, where working memory-known to have a great overlap with general cognition $^{54}$-was highlighted. Parkinsonism was the most central motor node possibly due to its higher construct complexity, capturing multiple subcomponents. Previous studies have shown that parkinsonism is a particularly important symptom in SSD for its high prevalence, spontaneous presence in drug-naïve patients, and association with cognition..$^{55}$

Regarding bridge strength centrality, 2 main sets of bridge nodes should be addressed. A first set was composed of metacognitive abilities - such as abstraction capacity (N5) and insight (G12) - the capacity to maintain attention (G11) and movement-related nodes (G7 and UPDRS). A careful look into the edges established by these bridge nodes also reveals proximity to the theory of mind (ToM) node and negative symptom nodes associated with the expressive deficit factor (N1 and N6). Taken together, these results highlight previous findings from different lines of research, for instance: the previously described association between 

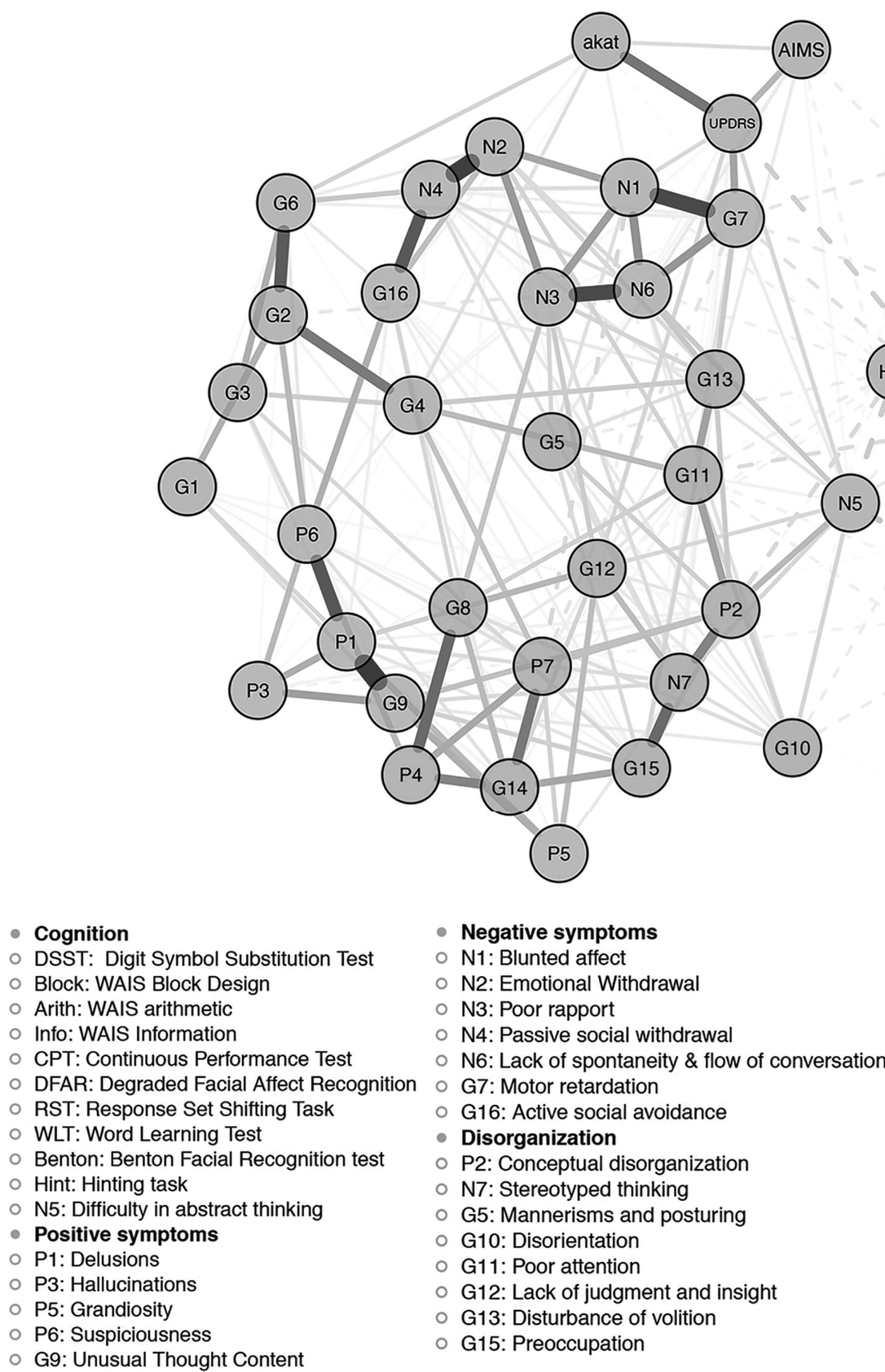

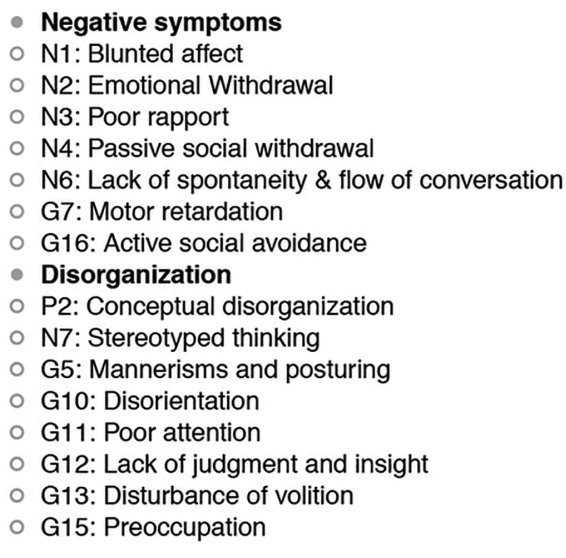

- Excitement

○ P7: Hostility

- P4: Excitement

○ G8: Uncooperativeness

- G14: Poor impulse control

- Emotional distress

- G1: Somatic concern

- G2: Anxiety

$\circ$ G3: Guilt feelings

$\circ$ G4: Tension

○ G6: Depression

- Motor Symptoms

- AIMS: Abnormal Involuntary Movement Scale

- UPDRS: Unified Parkinson Disease Rating Scale

$\circ$ akat: akathysia

Fig. 1. Estimated network $(N=1007)$. Nodes were grouped according to the community detection results using Exploratory Graph Analysis.

ToM, disorganization symptoms, and metaphor comprehension in $\mathrm{SSD}^{56,57}$; the relation between gestural perception and gestural performance, mediated by frontal lobe dysfunction, ${ }^{58}$ given that motor symptoms are associated with impaired nonverbal communication in psychosis $^{59}$; the association between parkinsonism and disorganization symptoms ${ }^{60}$; and the evidence that, in Parkinson's disease, conceptualization skills are particularly affected within frontal lobe functions. ${ }^{61}$ The fact that 2 of the 3 strongest bridge strength centrality nodes belonged to the disorganization community (G11 and G12) reiterates the pivoting role this community plays between psychopathology and cognition in line with findings from previous network analysis studies, including on high-risk subjects. ${ }^{17,19,52}$

A second set of bridging nodes was related to the "affective" components of the network and had "suspiciousness" as the hub, mostly binding positive symptoms, excitement, and emotional distress communities. This might indicate that suspiciousness is a bridge for the emergence of mood episodes within SSD. In a previous network analysis study, van Rooijen et $\mathrm{al}^{14}$ also found 

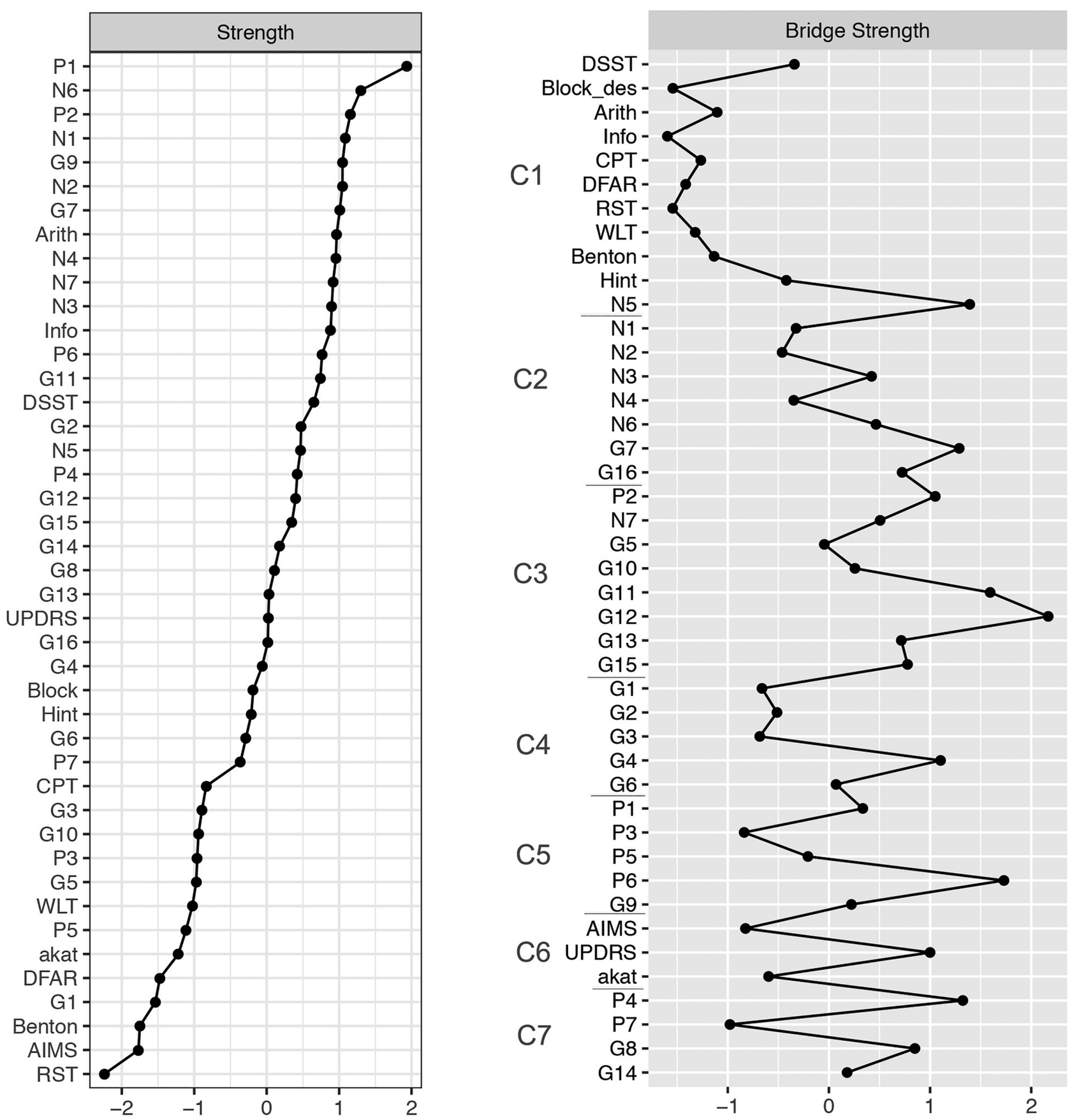

Fig. 2. Centrality indices plot (z-scores). Nodes in bridge strength centrality are ordered according to community placement (C1-7).

"suspiciousness" as relevant regarding co-occurrence with depressive symptoms, namely suicidality, in SSD.

\section{Network Comparisons of Subgroups}

The remission subsample's network showed a significantly lower density of connections. This might be caused by the reduction of self-reinforcing feedback loops that contribute to the maintenance of syndromes in line with the network theory of mental disorders. ${ }^{8}$ It could, however, be in part attributed to a floor-effect artifact. ${ }^{62}$
Network sparsity in remission was conspicuous in the subset of higher-order cognitive functions. This pattern mirrors previously described differences between patients and healthy subjects in terms of cognition factor structure - in patients, cognitive skills load more in one single " $\mathrm{g}$ " factor, implying that cognition is less domain specific than in controls. ${ }^{63}$

Moreover, network structure also differed with respect to remission status and, accordingly, node centrality hierarchy changed too. Interestingly, the highest disparities in centrality were not strictly found in nodes integrated 
A

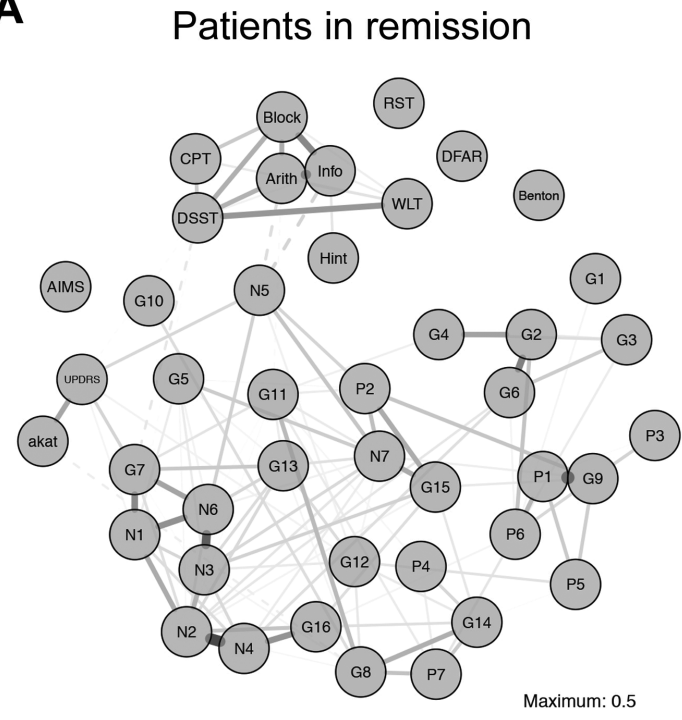

Patients not in remission

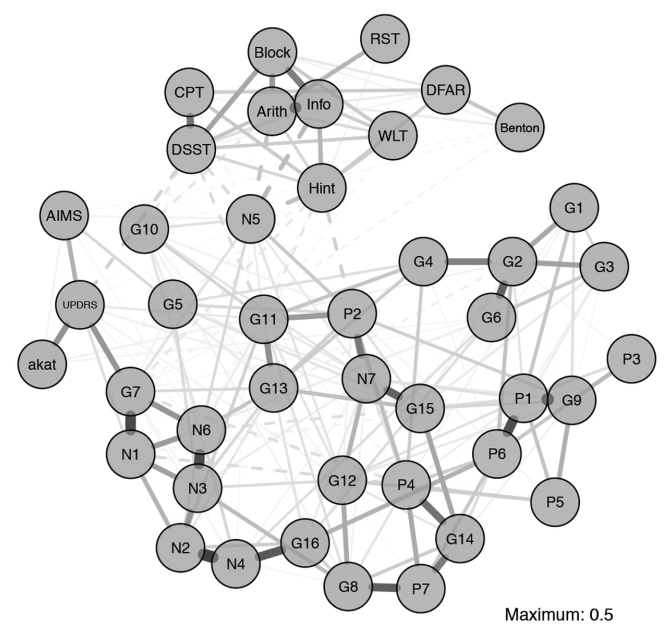

B

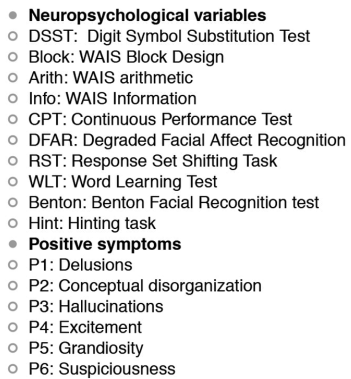

- DSST: Digit Symbol Substitution Test

- Block: WAIS Block Design

Arith: WAIS arithmetic

CPT: Continuous Performance Test

DFAR: Degraded Facial Affect Recognition

RST: Response Set Shifting Task

Benton: Benton Facial Recognition test

ting task

P1: Delusions

ual disorganization

P4: Excitement

P5: Grandiosity

P7: Hostility

Negative symptoms

- N1: Blunted affect

- N2: Emotional Withdrawal

N3: Poor rapport

N4: Passive social withdrawal

N5: Difficulty in abstract thinking

N6: Lack of spontaneity \& flow of conversation

N7: Stereotyped thinking

- General Symptoms

G2: Anxiety

G3: Guilt feeling

G4: Tension

G5: Mannerisms and posturing

G6: Depression

G7: Motor retardation

G8: Uncooperativeness

G9: Unusual Though

G11: Poor attention

G12: Lack of judgment and in
G13: Disturbance of volition

- G14: Poor impulse control

- G15: Preoccupation

$\circ$ G15: Preoccupation
$\circ$ G16: Active social avoidance

- Motor symptoms

AIMS: Abnormal Involuntary Movement Scale

UPDRS: Unified Parkinson Disease Rating Scale

$\circ$ akat: akathysia
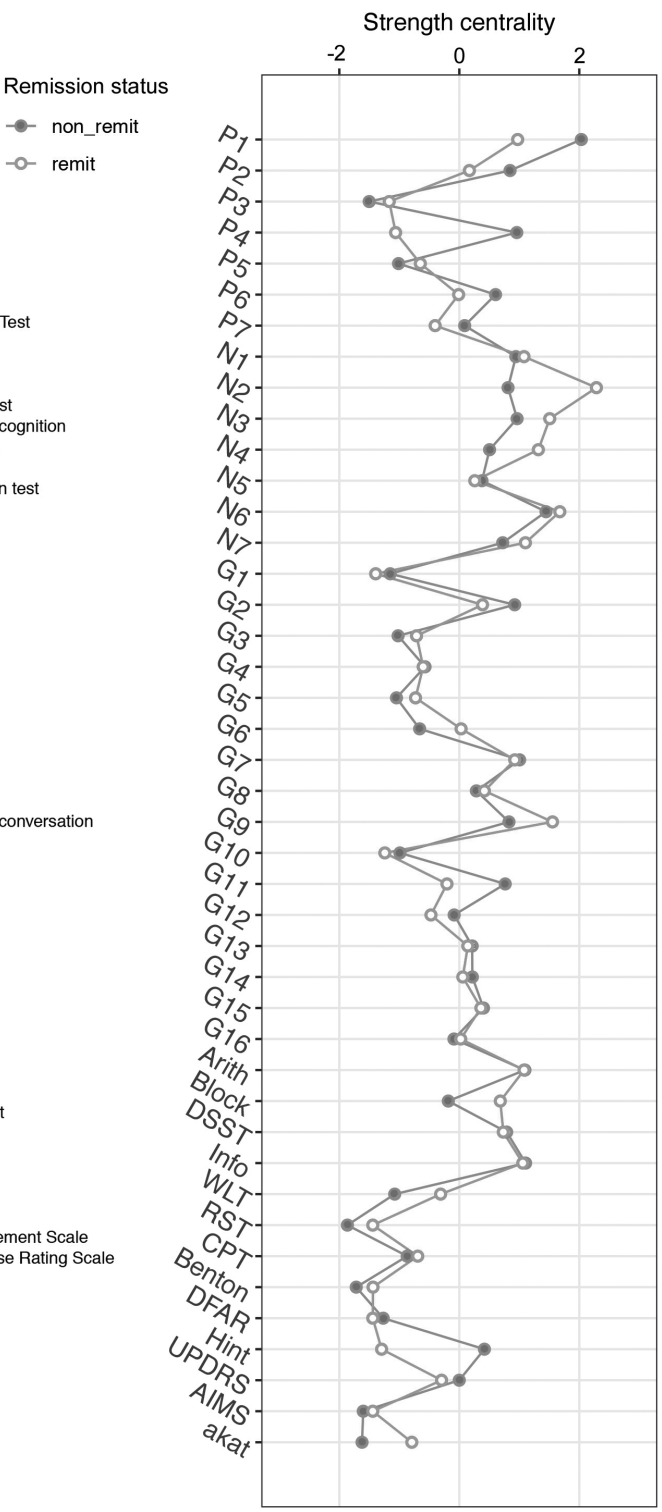

Fig. 3. Network comparison according to remission status. A, network layouts; B, strength centrality indices (z-score) per remission status.

into remission criteria (eg, P4 and N2). Nodes commonly associated with the avolition factor were more central in remission. This is in line with studies that show that the avolition factor is less state dependent and that other negative symptoms improve more during stabilization. ${ }^{64}$ Other network analysis studies showed avolition to be a very central negative symptom and a particularly important one to target when it comes to the treatment of this symptom domain. ${ }^{65,66}$ This finding should, however, be read cautiously as avolition is poorly defined by the PANSS.

These results comply with another network analysis study comprising cognitive nodes, which also found different network density and structure between recovered and nonrecovered patients with schizophrenia. ${ }^{67}$ However, they partially concur ${ }^{14}$ or contradict ${ }^{68} 2$ previous studies. These discrepancies might be explained by differences in the included nodes in one case and significant methodological divergences in the other.

On a cautionary note, 2 aspects limit the interpretation of these findings. First, due to smaller sample size, the estimated networks for each group are less stable, hence possibly failing more to correctly estimate the true edges. Second, these findings might partly be explained by sociodemographical and clinical differences between the 2 subsamples. 


\section{Strengths and Limitations}

Some of this study's strengths flow from the innovative network approach to psychiatric disorders and include: the joint analysis of many variables, hence dissecting the correlational architecture of the disorder at a system level, and the use of metrics that are specific to this approach and that allow for exploration of results in an objective fashion. In addition, this study benefitted from a large sample size; a thorough stability analysis that corroborated the reliability of results; and the inclusion of a broad set of patients - diagnoses along the nonaffective psychosis spectrum (not limited to schizophrenia) and both males and females.

A first limitation of this study is its group-level approach; thus, extrapolations to the individual level should be performed carefully. Studies using longitudinal data could tackle this limitation in the future. In addition, the cross-sectional nature of the data precludes causal interpretation of associations. Second, important elements might be lacking from the cognitive and motor domains (eg, visuo-spatial memory or neurological soft signs) and, concerning the latter, the inclusion of more objective measures (eg, actigraphy) could have reduced bias caused by poorly defined variables (eg, "G7-motor retardation"). ${ }^{69}$ Third, a node representing antipsychotic medication was not included due to a lack of data on the prescribed dosages. However, the available information on the type of medication used and a further sensitivity analysis showed that only a minority of patients was taking typical antipsychotics and that this variable did not seem to affect the network's connectivity.

\section{Conclusions}

In this study, we showed that cognitive and motor symptoms also played an important role in a network of core clinical variables of SSD. Moreover, we found that a group of metarepresentational abilities (abstraction, insight, and theory of mind), expressive deficit negative symptoms, and motor disturbances composed an important aggregating set of symptoms. We believe that these findings have implications (1) for phenomenology and cognitive science in that they reiterate the fundamental role of motor behavior and specific higher-order skills, respectively; (2) for neuroscientific research, encouraging research on brain systems that integrate cognitive, motor, and affective functions ${ }^{70}$; and, eventually, (3) for treatment approaches, emboldening metacognitive and embodied therapies as they target what seem to be the most unifying elements of the estimated network. Lastly, our findings endorse the integration of remission status into future network analysis studies of mental disorders with oscillatory dynamics (eg, SSD and mood disorders).

\section{Supplementary Material}

Supplementary material is available at Schizophrenia Bulletin.

\section{Acknowledgments}

We are grateful for the generosity of time and effort by the patients, their families, and healthy subjects. Furthermore, we would like to thank all research personnel involved in the GROUP project, in particular, Joyce van Baaren, Erwin Veermans, Ger Driessen, Truda Driesen, and Erna van't Hag. B.M.M. would like to thank Alessia Avila for suggestions and proofreading.

\section{Funding}

This work was supported by Fundação para a Ciência e Tecnologia (grant number PD/BD/128404/2017). The infrastructure for the GROUP study is funded through the Geestkracht programme of the Dutch Health Research Council (Zon-Mw, grant number 10-000-1001), and matching funds from participating pharmaceutical companies (Lundbeck, AstraZeneca, Eli Lilly, and Janssen Cilag) and universities and mental health care organizations (Amsterdam: Academic Psychiatric Centre of the Academic Medical Center and the mental health institutions GGZ Ingeest, Arkin, Dijk en Duin, GGZ Rivierduinen, Erasmus Medical Centre, GGZ Noord Holland Noord. Groningen: University Medical Center Groningen and the mental health institutions Lentis, GGZ Friesland, GGZ Drenthe, Dimence, Mediant, GGNet Warnsveld, Yulius Dordrecht and Parnassia psychomedical center The Hague. Maastricht: Maastricht University Medical Centre and the mental health institutions GGzE, GGZ Breburg, GGZ Oost-Brabant, Vincent van Gogh voor Geestelijke Gezondheid, Mondriaan, Virenze riagg, Zuyderland GGZ, MET ggz, Universitair Centrum Sint-Jozef Kortenberg, CAPRI University of Antwerp, PC Ziekeren Sint-Truiden, PZ Sancta Maria Sint-Truiden, GGZ Overpelt, OPZ Rekem. Utrecht: University Medical Center Utrecht and the mental health institutions Altrecht, GGZ Centraal and Delta).

\section{Genetic Risk and Outcome of Psychosis (GROUP) Investigators}

\section{Therese van Amelsvoort ${ }^{1}$, Agna A. Bartels- Velthuis $^{2}$, Richard Bruggeman ${ }^{2,3}$, Wiepke Cahn ${ }^{4,5}$, Lieuwe de Haan ${ }^{6,7}$, Rene S. Kahn ${ }^{8,9}$, and Claudia J. P. Simons ${ }^{1,10}$}

${ }^{1}$ Department of Psychiatry and Neuropsychology, School for Mental Health and Neuroscience, Maastricht University Medical Center, Maastricht, The Netherlands; ${ }^{2}$ Rob Giel Research Center, University Center for Psychiatry, University Medical 
Center Groningen, University of Groningen, Groningen, The Netherlands; ${ }^{3}$ Department of Clinical and Developmental Neuropsychology, University of Groningen, Groningen, The Netherlands; ${ }^{4}$ Department of Psychiatry, Brain Centre Rudolf Magnus, University Medical Center Utrecht, Utrecht University, Utrecht, The Netherlands; ${ }^{5}$ General Mental Health Care,

Altrecht, Utrecht, The Netherlands; ${ }^{6}$ Department of Psychiatry, Amsterdam UMC, University of Amsterdam, Amsterdam, The Netherlands; ${ }^{7}$ Arkin Institute for Mental Health, Amsterdam, The Netherlands; ${ }^{8}$ Department of Translational Neuroscience, Brain Center Rudolf Magnus, University Medical Center Utrecht, Utrecht, The Netherlands; ' Department of Psychiatry, Icahn School of Medicine at Mount Sinai, New York, NY, USA; ${ }^{10} \mathrm{GGzE}$ Institute for Mental Health Care, Eindhoven, The Netherlands

\section{References}

1. Kirkpatrick B. Schizophrenia as a systemic disease. Schizophr Bull. 2009;35(2):381-382.

2. Couture SM, Granholm EL, Fish SC. A path model investigation of neurocognition, theory of mind, social competence, negative symptoms and real-world functioning in schizophrenia. Schizophr Res. 2011;125(2-3):152-160.

3. Green MF. Impact of cognitive and social cognitive impairment on functional outcomes in patients with schizophrenia. J Clin Psychiatry. 2016;77(suppl 2):8-11.

4. Mittal VA, Walther S. As motor system pathophysiology returns to the forefront of psychosis research, clinical implications should hold center stage. Schizophr Bull. 2019;45(3):495-497.

5. van Harten PN, Walther S, Kent JS, Sponheim SR, Mittal VA. The clinical and prognostic value of motor abnormalities in psychosis, and the importance of instrumental assessment. Neurosci Biobehav Rev. 2017;80:476-487.

6. Cuesta MJ, Moreno-Izco L, Ribeiro M, et al. Motor abnormalities and cognitive impairment in first-episode psychosis patients, their unaffected siblings and healthy controls. Schizophr Res. 2018;200:50-55.

7. Strik W, Stegmayer K, Walther S, Dierks T. Systems neuroscience of psychosis: mapping schizophrenia symptoms onto brain systems. Neuropsychobiology. 2017;75(3): $100-116$.

8. Borsboom D. A network theory of mental disorders. World Psychiatry. 2017;16(1):5-13.

9. Fried EI, van Borkulo CD, Cramer AO, Boschloo L, Schoevers RA, Borsboom D. Mental disorders as networks of problems: a review of recent insights. Soc Psychiatry Psychiatr Epidemiol. 2017;52(1):1-10.

10. Borsboom D, Cramer AO. Network analysis: an integrative approach to the structure of psychopathology. Annu Rev Clin Psychol. 2013;9:91-121.

11. Greene JA, Loscalzo J. Putting the patient back togethersocial medicine, network medicine, and the limits of reductionism. N Engl J Med. 2017;377(25):2493-2499.

12. Robinaugh DJ, Hoekstra RHA, Toner ER, Borsboom D. The network approach to psychopathology: a review of the literature 2008-2018 and an agenda for future research. Psychol Med. 2020;50(3):353-366.

13. Epskamp S, Waldorp LJ, Mõttus R, Borsboom D. The Gaussian graphical model in cross-sectional and time-series data. Multivariate Behav Res. 2018;53(4):453-480.

14. van Rooijen G, Isvoranu AM, Kruijt $\mathrm{OH}$, et al.; GROUP investigators. A state-independent network of depressive, negative and positive symptoms in male patients with schizophrenia spectrum disorders. Schizophr Res. 2018;193: 232-239.

15. van Rooijen G, Isvoranu AM, Meijer CJ, van Borkulo CD, Ruhé HG, de Haan L; GROUP investigators. A symptom network structure of the psychosis spectrum. Schizophr Res. 2017; 189:75-83.

16. Isvoranu AM, van Borkulo CD, Boyette LL, Wigman JT, Vinkers $\mathrm{CH}$, Borsboom D; Group Investigators. A network approach to psychosis: pathways between childhood trauma and psychotic symptoms. Schizophr Bull. 2017;43(1):187-196.

17. Chang WC, Wong CSM, Or PCF, et al. Inter-relationships among psychopathology, premorbid adjustment, cognition and psychosocial functioning in first-episode psychosis: a network analysis approach. Psychol Med. 50(12):2019:1-9.

18. Galderisi S, Rucci P, Kirkpatrick B, et al.; Italian Network for Research on Psychoses. Interplay among psychopathologic variables, personal resources, contextrelated factors, and real-life functioning in individuals with schizophrenia: a network analysis. JAMA Psychiatry. 2018;75(4):396-404.

19. Hasson-Ohayon I, Goldzweig G, Lavi-Rotenberg A, Luther L, Lysaker PH. The centrality of cognitive symptoms and metacognition within the interacting network of symptoms, neurocognition, social cognition and metacognition in schizophrenia. Schizophr Res. 2018;202:260-266.

20. Korver N, Quee PJ, Boos HB, Simons CJ, de Haan L; GROUP investigators. Genetic Risk and Outcome of Psychosis (GROUP), a multi-site longitudinal cohort study focused on gene-environment interaction: objectives, sample characteristics, recruitment and assessment methods. Int J Methods Psychiatr Res. 2012;21(3): 205-221

21. American Psychiatric Association. Diagnostics and Statistical Manual of Mental Disorders. 4th ed., text rev. ed. Washington, DC:American Psychiatric Association; 2000.

22. Kay SR, Fiszbein A, Opler LA. The positive and negative syndrome scale (PANSS) for schizophrenia. Schizophr Bull. 1987;13(2):261-276.

23. Nuechterlein KH, Barch DM, Gold JM, Goldberg TE, Green MF, Heaton RK. Identification of separable cognitive factors in schizophrenia. Schizophr Res. 2004;72(1):29-39.

24. Weschler D. WAIS-III: Wechsler Adult Intelligence Scale, Administration and Scoring Manual. San Antonio, TX: Psychological Corporation, Harcourt Brace; 1997.

25. Bilder RM, Turkel E, Lipschutz-Broch L, Lieberman JA. Antipsychotic medication effects on neuropsychological functions. Psychopharmacol Bull. 1992;28(4):353-366.

26. Brand N, Jolles J. Learning and retrieval rate of words presented auditorily and visually. J Gen Psychol. 1985;112(2):201-210.

27. Nuechterlein KH, Dawson ME. Information processing and attentional functioning in the developmental course of schizophrenic disorders. Schizophr Bull. 1984;10(2):160-203.

28. Benton AL. Sivan AB, de Hamsher KS, Varney NR, Spreen O. Benton's Test of Facial Recognition. New York: Oxford University Press; 1983.

29. van't Wout M, Aleman A, Kessels RP, Laroi F, Kahn RS. Emotional processing in a non-clinical psychosis-prone sample. Schizophr Res. 2004;68(2-3):271-281. 
30. Corcoran R, Mercer G, Frith CD. Schizophrenia, symptomatology and social inference: investigating "theory of mind" in people with schizophrenia. Schizophr Res. 1995;17(1):5-13.

31. Martínez-Martín P, Gil-Nagel A, Gracia LM, Gómez JB, Martínez-Sarriés J, Bermejo F. Unified Parkinson's Disease Rating Scale characteristics and structure. The Cooperative Multicentric Group. Mov Disord. 1994;9(1):76-83.

32. Barnes TRE. A rating scale for drug-induced akathisia. $\mathrm{Br} J$ Psychiatry. 1989;154(05):672-676.

33. Guy WA. Abnormal Involuntary Movement Scale (AIMS). Washington, DC: Department of Health Education and Welfare; 1976.

34. Buuren Sv, Groothuis-Oudshoorn K. mice: multivariate imputation by chained equations inR. J Stat Softw. 2011;45(3):1-67.

35. Liu H, Lafferty J, Wasserman L. The nonparanormal: semiparametric estimation of high dimensional undirected graphs. J Mach Learn Res. 2009;10:2295-2328.

36. R Core Team. R: A Language and Environment for Statistical Computing. Vienna, Austria: R Foundation for Statistical Computing; 2013. http://www.R-project.org. Accessed September 1, 2020.

37. Epskamp S, Borsboom D, Fried EI. Estimating psychological networks and their accuracy: A tutorial paper. Behav Res Meth. 2018;50(1):195-212.

38. Epskamp S, Cramer AOJ, Waldorp LJ, Schmittmann VD, Borsboom D. qgraph: Network visualizations of relationships in psychometric data. J Stat Softw. 2012;48(4):1-18.

39. Foygel R, Drton M. Extended Bayesian information criteria for Gaussian graphical models. Adv Neural Inf Process Syst. 2010;1:604-612.

40. Fruchterman TMJ, Reingold EM. Graph drawing by force-directed placement. Softw Pract Exp. 1991;21(11): 1129-1164.

41. Golino HF, Epskamp S. Exploratory graph analysis: a new approach for estimating the number of dimensions in psychological research. PLoS One. 2017;12(6):e0174035.

42. Christensen AP, Golino H. Estimating the Stability of the Number of Factors via Bootstrap Exploratory Graph Analysis: A Tutorial. 2019. doi:10.31234/osf.io/9deay

43. Humphries MD, Gurney K. Network "small-world-ness": a quantitative method for determining canonical network equivalence. PLoS One. 2008;3(4):e0002051.

44. Jones PJ. networktools: Tools for identifying important nodes in networks. R package version 12. 2018. https://CRAN.Rproject.org/package=networktools. Accessed September 1, 2020.

45. Jones PJ, Ma R, McNally RJ. Bridge centrality: a network approach to understanding comorbidity. Multivariate Behav Res. 2019:1-15.

46. Andreasen NC, Carpenter WT Jr, Kane JM, Lasser RA, Marder SR, Weinberger DR. Remission in schizophrenia: proposed criteria and rationale for consensus. $A m J$ Psychiatry. 2005;162(3):441-449.

47. van Borkulo CD, Boschloo L, Kossakowski JJ, et al. Comparing network structures on three aspects: a permutation test. 2016;34. doi:10.13140/RG.2.2.29455.38569

48. Wallwork RS, Fortgang R, Hashimoto R, Weinberger DR, Dickinson D. Searching for a consensus five-factor model of the Positive and Negative Syndrome Scale for schizophrenia. Schizophr Res. 2012;137(1-3):246-250.
49. Borsboom D, Cramer AO, Schmittmann VD, Epskamp S, Waldorp LJ. The small world of psychopathology. PLoS One. 2011;6(11):e27407.

50. van der Gaag M, Hoffman T, Remijsen M, et al. The fivefactor model of the Positive and Negative Syndrome Scale II: a ten-fold cross-validation of a revised model. Schizophr Res. 2006;85(1-3):280-287.

51. Hallquist MN, Wright AGC, Molenaar PCM. Problems with centrality measures in psychopathology symptom networks: why network psychometrics cannot escape psychometric theory. Multivariate Behav Res. 2019:1-25.

52. Jimeno N, Gomez-Pilar J, Poza J, et al. Main symptomatic treatment targets in suspected and early psychosis: new insights from network analysis. Schizophr Bull. 2020;46(4):884-895.

53. Levine SZ, Leucht S. Identifying a system of predominant negative symptoms: network analysis of three randomized clinical trials. Schizophr Res. 2016;178(1-3):17-22.

54. Colom R. Working memory is (almost) perfectly predicted by g. Intelligence. 2004;32(3):277-296.

55. Cuesta MJ, Sánchez-Torres AM, de Jalón EG, et al. Spontaneous parkinsonism is associated with cognitive impairment in antipsychotic-naive patients with first-episode psychosis: a 6-month follow-up study. Schizophr Bull. 2014;40(5):1164-1173.

56. de Sousa P, Sellwood W, Griffiths M, Bentall RP. Disorganisation, thought disorder and socio-cognitive functioning in schizophrenia spectrum disorders. Br J Psychiatry. 2019;214(2):103-112.

57. Gavilán JM, García-Albea JE. Theory of mind and language comprehension in schizophrenia: poor mindreading affects figurative language comprehension beyond intelligence deficits. J Neurolinguist. 2011;24(1):54-69.

58. Walther S, Stegmayer K, Sulzbacher J, et al. Nonverbal social communication and gesture control in schizophrenia. Schizophr Bull. 2015;41(2):338-345.

59. Dutschke LL, Stegmayer K, Ramseyer F, et al. Gesture impairments in schizophrenia are linked to increased movement and prolonged motor planning and execution. Schizophr Res. 2018;200:42-49.

60. Cortese L, Caligiuri MP, Malla AK, Manchanda R, Takhar J, Haricharan R. Relationship of neuromotor disturbances to psychosis symptoms in first-episode neuroleptic-naive schizophrenia patients. Schizophr Res. 2005;75(1):65-75.

61. Lima CF, Meireles LP, Fonseca R, Castro SL, Garrett C. The Frontal Assessment Battery (FAB) in Parkinson's disease and correlations with formal measures of executive functioning. $J$ Neurol. 2008;255(11):1756-1761.

62. Terluin B, de Boer MR, de Vet HC. Differences in connection strength between mental symptoms might be explained by differences in variance: reanalysis of network data did not confirm staging. PLoS One. 2016;11(11):e0155205.

63. Dickinson D, Ragland JD, Calkins ME, Gold JM, Gur RC. A comparison of cognitive structure in schizophrenia patients and healthy controls using confirmatory factor analysis. Schizophr Res. 2006;85(1-3):20-29.

64. Üçok A, Ergül C. Persistent negative symptoms after first episode schizophrenia: a 2-year follow-up study. Schizophr Res. 2014;158(1-3):241-246.

65. Strauss GP, Esfahlani FZ, Galderisi S, et al. Network analysis reveals the latent structure of negative symptoms in schizophrenia. Schizophr Bull. 2019;45(5):1033-1041.

66. Strauss GP, Zamani Esfahlani F, Sayama H, et al. Network analysis indicates that avolition is the most central domain 
for the successful treatment of negative symptoms: evidence from the Roluperidone randomized clinical trial. Schizophr Bull. 2020;46(4):964-970.

67. Galderisi S, Rucci P, Mucci A, et al.; Italian Network for Research on Psychoses. The interplay among psychopathology, personal resources, context-related factors and reallife functioning in schizophrenia: stability in relationships after 4 years and differences in network structure between recovered and non-recovered patients. World Psychiatry. 2020;19(1):81-91.
68. Esfahlani FZ, Sayama H, Visser KF, Strauss GP. Sensitivity of the Positive and Negative Syndrome Scale (PANSS) in detecting treatment effects via network analysis. Innov Clin Neurosci. 2017;14(11-12):59-67.

69. Walther S, Koschorke P, Horn H, Strik W. Objectively measured motor activity in schizophrenia challenges the validity of expert ratings. Psychiatry Res. 2009;169(3):187-190.

70. Mittal VA, Bernard JA, Northoff G. What can different motor circuits tell us about psychosis? An RDoC perspective. Schizophr Bull. 2017;43(5):949-955. 